\title{
ESPÉCIES REATIVAS DO OXIGÊNIO E DOENÇA RENAL CRÔNICA: REVISÃO DE LITERATURA
}

\author{
GALVÃO, André Luiz Baptista ${ }^{1}$ \\ PALAZZO, Elzylene Léga ${ }^{2}$ \\ PINTO, Mildre Loraine ${ }^{3}$ \\ VIEIRA, Manuela Cristina ${ }^{4}$
}

\begin{abstract}
RESUMO: Quando se analisa o mecanismo fisiopatológico básico dos transtornos renais, observa-se a existência de fatores que predispõem ao desequilíbrio oxidativo. A instalação do estresse oxidativo decorre da existência de um desequilíbrio entre compostos oxidantes e antioxidantes, favorecendo a geração e acúmulo de espécies reativas do oxigênio (ERO). Tal evento conduz à oxidação de biomoléculas com consequente perda de funções biológicas e/ou desequilíbrio homeostático, cuja manifestação é o dano oxidativo potencial contra células e tecidos. A hipertensão arterial sistêmica (HAS), inflamação e disfunção endotelial que estão relacionadas no desenvolvimento de doenças cardiovasculares e proteinúria são implicadas como consequências do estresse oxidativo na progressão da doença renal crônica (DRC). Animais com DRC têm, frequentemente, condições concorrentes que aumentam a geração das ERO, tais como idade avançada, ativação do sistema renina-angiotensina (SRA) e desordens sistêmicas diversas. A existência de anemia nestes pacientes exacerba o problema em razão da redução da ação antioxidante renal normalmente exercida pelos eritrócitos. Descrever a participação das ERO na fisiopatologia da DRC trata-se do objetivo da presente revisão de literatura.
\end{abstract}

Palavras-chave: Estresse. Oxidação. Radicais-livres.

\section{REACTIVE OXYGEN SPECIES AND CHRONIC KIDNEY DISEASE: REVIEW}

\begin{abstract}
SUMMARY: When analyzing the basic pathophysiological mechanism of kidney disorders, notes the existence of factors that predispose to oxidative imbalance. The installation of oxidative stress results from an imbalance between oxidants and antioxidants compounds, favoring the generation and accumulation of reactive oxygen species (ROS) . This event leads to the oxidation of biomolecules with consequent loss of biological function and/or homeostatic imbalances whose expression is oxidative damage to cells and tissues against potential. High blood pressure (HBP), inflammation and endothelial dysfunction are related to the development of cardiovascular disease and proteinuria are implicated consequences of oxidative stress in the progression of chronic kidney disease (CKD). Animals with CKD often have concurrent conditions that increase the generation of ROS, such as advanced age, activation of the renin - angiotensin system (RAS) and various systemic disorders. The presence of anemia in these patients exacerbates the problem because of reduced renal antioxidant normally exercised by erythrocytes. Describe the involvement of ROS in the pathophysiology of CKD it is the purpose of this literature review.
\end{abstract}

Keywords: Stress. Oxidation. Free radicals.

\section{INTRODUÇÃO}

O estresse oxidativo ocorre quando a produção de espécies reativas do oxigênio (ERO)

\footnotetext{
${ }^{1}$ Doutorando em Medicina Veterinária pela Faculdade de Ciências Agrárias e Veterinárias Universidade Estadual Paulista campus Jaboticabal (SP). Email: andrelgalvao@ hotmail.com.

${ }^{2}$ Docente da Faculdade Dr. Francisco Maeda - Fundação Educacional de Ituverava (SP).

${ }^{3}$ Docente do Centro Universitário Moura Lacerda; Centro Universitário Barão de Mauá, Ribeirão Preto (SP).

${ }^{4}$ Doutora em Medicina Veterinária da Faculdade de Ciências Agrárias e Veterinárias Universidade Estadual Paulista campus Jaboticabal (SP).
} 
ultrapassam a capacidade de defesa do sistema antioxidante, resultando em dano tecidual (CACHOFEIRO et al., 2008). A geração de ERO é resultante do metabolismo de oxigênio. A mitocôndria, por meio da cadeia transportadora de elétrons, é a principal fonte geradora. $\mathrm{O}$ sistema de defesa antioxidante tem a função de inibir e/ou minimizar os efeitos deletérios provocados pela ação das ERO (BARBOSA et al., 2010). Acredita-se que o estresse oxidativo seja um fator potencialmente importante na mortalidade dos pacientes com doença renal crônica (DRC) e na mediação de muitas complicações, pois está envolvido na patogênese da hipertensão arterial sistêmica (HAS), disfunção endotelial e inflamação (SCOTT, 2008; URSO; CAIMI, 2011; ZATZ et al., 2012).

As mais importantes ERO incluem: o ânion superóxido $\left(\mathrm{O}_{2}^{-}\right)$, peróxido de hidrogênio $\left(\mathrm{H}_{2} \mathrm{O}_{2}\right)$ e radical hidroxila $\left(\mathrm{OH}^{-}\right)$(BARBOSA et al., 2010). $\mathrm{O} \mathrm{O}_{2}{ }^{-}$, é produzido continuamente por diversos processos celulares como na cadeia de transporte de elétrons na mitocôndria, no microssomo, através de enzimas como xantina oxidase e nicotinamida adenina dinucleotídeo fosfato (NADPH oxidase), ou pela redução monoelétrica de $\mathrm{O}_{2} \cdot \mathrm{O} \mathrm{H}_{2} \mathrm{O}_{2}$ é formado pela reação da dismutação de $\mathrm{O}_{2}{ }^{-}$catalizada pela enzima superóxido dismutase (SOD), pela redução de $2 \mathrm{e}^{-}$na molécula de $\mathrm{O}_{2}$, tratando-se de uma molécula muito difusível entre as células. $\mathrm{O} \mathrm{OH}^{-}$é o mais reativo de todos os radicais livres e o mais lesivo radical conhecido, uma vez formado, causa modificações no DNA, danos nas proteínas e inativação enzimática com consequente peroxidação lipídica (VASCONCELOS et al., 2007; BARBOSA et al., 2010).

Também são considerados como ERO: (a) o ácido hipocloroso (HOCl) que oxida um grande número de compostos biológicos, como tióis e tioésteres, aminas, fenóis e ligações insaturadas, sendo mais seletivo que o radical hidroxila, oxida ferro e proteínas; (b) os lipídios, em que a peroxidação lipídica é causada pelo ação de uma ERO, geralmente $\mathrm{OH}^{-}$, que abstrai um átomo de hidrogênio $\left(\mathrm{H}^{+}\right)$de um grupo metileno alílico, normalmente de um ácido graxo poliinsaturado, deixando um elétron desemparelhado de carbono, caracterizando a etapa de iniciação; (c) o peróxido nitrito (ONOO-) trata-se de um radical livre instável, potente, com propriedades similares ao radical $\mathrm{OH}^{-}$e causa danos a muitas moléculas biológicas (VASCONCELOS et al., 2007)

Frente à ação potencialmente lesiva promovida pelas ERO, o organismo possui o sistema de defesa antioxidante. Tais ações podem ser alcançadas por meio de diferentes mecanismos de ação como: inibindo a formação das ERO, impedindo a ação das ERO e favorecendo o reparo e a reconstituição das estruturas biológicas lesadas (sistemas de reparo) (BARBOSA et al., 2010).

O sistema antioxidante é dividido em enzimático e não enzimático. No sistema enzimático estão inclusos as enzimas SOD, catalase (CAT), glutationa peroxidase (GPx). Essas enzimas agem por meio de mecanismos de prevenção, impedindo e/ou controlando a formação de ERO, 
envolvidos com a iniciação das reações em cadeia que culminam com a propagação e amplificação do processo e, consequentemente, com a ocorrência de danos oxidativos (VASCONCELOS et al., 2007; BARBOSA et al., 2010). No sistema não-enzimático estão inclusos os compostos antioxidantes de origem dietética, entre os quais se destacam: vitaminas, minerais e compostos fenólicos. O ácido ascórbico (vitamina $\mathrm{C}$ ), o $\alpha$-tocoferol e $\beta$-caroteno, precursores da vitamina $\mathrm{E}$ e $\mathrm{A}$, respectivamente, são compostos vitamínicos potencialmente antioxidantes. São ainda considerados importantes antioxidantes os carotenóides sem a atividade da vitamina A, como licopeno, luteína e zeaxantina. Entre os minerais destacam-se o zinco, cobre, selênio e magnésio (BARBOSA et al., 2010).

Considerando a potencialidade da ação do radical $\mathrm{OH}^{-}$, é de extrema importância a manutenção do perfeito equilíbrio entre as enzimas antioxidantes, com o propósito de promover a manutenção da integridade celular. Desse modo, merece destaque a ação da GPx, uma vez que sua ação depende da manutenção do ciclo redox da glutationa, por meio do controle da relação entre glutationa reduzida (GSH) e glutationa oxidada (GSSG) (BARBOSA et al. 2010). Descrever a importância das ERO na fisiopaotlogia da DRC é o objetivo da presente revisão de literatura.

\section{COMPROMETIMENTO DA FUNÇÃO RENAL}

O paciente com transtornos renais, geralmente, apresenta-se mal nutrido, com carências em reservas de vitaminas e minerais, o que diminui os mecanismos de defesa antioxidante e favorece a instalação do estresse oxidativo (LOCATELLI et al., 2003). Nestas condições, consideram-se as implicações da síndrome urêmica (anorexia, vômitos, paladar seletivo e gastrite) concomitante à dieta restrita, à má absorção de nutrientes e às perdas urinárias de vitaminas hidrossolúveis e proteína (WILCOX; TISHER, 2008).

No parênquima renal as células glomerulares, células tubulares e os macrófagos são geradores potenciais de ERO (GALLE, 2001). As ERO com ações importantes nos rins incluem o $\mathrm{O}_{2}{ }^{-}, \mathrm{H}_{2} \mathrm{O}_{2}, \mathrm{OH}^{-}, \mathrm{HOCl}$, lipídios, ONOO-, entre outros (LOCATELLI et al., 2003).

Animais com DRC têm, frequentemente, condições concorrentes que aumentam a geração das ERO, tais como idade avançada, ativação do sistema renina angiotensina aldosterona (SRAA) e desordens sistêmicas diversas. A presença de fibrose intersticial no parênquima renal resulta em deficiência na atividade mitocondrial local, promovendo, assim, a geração das ERO (SCOTT 2008).

A redução da taxa de filtração glomerular (TFG) na evolução da DRC em cães e gatos constitui um fator para a instalação do estresse oxidativo no rim. Em condições normais é 
produzida uma variedade de ERO dentro das células renais, como resultante do metabolismo aeróbico, porém, na vigência de DRC ocorre aumento das ERO. Na DRC, caracteristicamente, ocorre hipertrofia e hipertensão glomerular dos néfrons remanescentes, o que pode, mesmo que temporariamente, manter a TFG. Este processo resulta em incremento da fosforilação oxidativa celular renal e aumento das ERO potencialmente lesivas. Em adição, os sistemas de defesa antioxidantes dos doentes renais crônicos são relativamente deficientes (SCOTT, 2008). A ativação crônica de substâncias oxidativas, tal qual ocorre na uremia, atinge proporções patológicas, contribuindo para dano celular e tecidual sistêmico (LOCATELLI et al., 2003; CAMPBELL, 2013).

Segundo Shimizu (2005) e Syme et al. (2007), a HAS contribui significativamente para a morbidade cardiovascular e mortalidade dos pacientes renais crônicos e está diretamente relacionada com o SRAA e também com a peroxidação lipídica. De acordo com Scoot (2008), a disfunção endotelial dos vasos sanguíneos é caracterizada por alterações na capacidade de dilatação vascular, decorrente da deficiência de óxido nítrico (NO), que, por sua vez, promove vasoconstrição e aderência de moléculas tais como VCAM-1 (molécula de adesão vascular), ICAM-1 (molécula de adesão intercelular) e P e E-selectina. Estas moléculas servem como receptores de leucócitos circulantes nas artérias, que são atraídos por meio da transcrição do fator nuclear Kappa $\beta$ (NF- $\kappa \beta)$, TNF- $\alpha$ (fator de necrose tumoral - alfa) e IL-1, estabelecendo assim o processo inflamatório e a hipertensão arterial (ZAFARULLAH et al., 2003; SCOTT, 2008; URSO; CAIMI, 2011; BROWN, 2013; CAMPBELL, 2013).

A ativação do SRAA desempenha um papel importante no desenvolvimento e na fisiopatologia de doenças do sistema cardiovascular, em especial na HAS e DRC (SAVOIA; SCHIFFRIN, 2006). A angiotensina II (AII) atua como potente agente pró-inflamatório, modulando repostas com quimiotaxia, proliferação e diferenciação de monócitos em macrófagos, através do aumento da liberação de moléculas de adesão e citocinas, induzindo a aderência de células inflamatórias às células endoteliais. Este peptídeo promove vasoconstrição da arteríola eferente glomerular, que reduz o fluxo sanguíneo e aumenta a filtração glomerular, contribuindo para o acréscimo do estresse oxidativo no néfron (SCOTT, 2008).

A interação da AII e seu receptor AT1 modulam a resposta inflamatória envolvida com estresse oxidativo, podendo promover efeitos cronotrópicos e inotrópicos positivos no coração, induzir hipertrofia e apoptose das células cardíacas. A administração da AII por via subcutânea em camundongos demonstrou hipertrofia da camada média da artéria aorta e infiltração perivascular por linfócitos $\mathrm{T}$ e macrófagos, com consequente espessamento da parede vascular, bem como lesão aos miócitos e fibrose intersticial em células cardíacas podem estar presentes. 
Entretanto, quando administrada pela via intravenosa a AII pode estar relacionada com a HAS, lesão glomerular, proteinúria e fibrose intersticial no tecido renal (CAMPBELL, 2013).

Alguns autores referiram que a administração de AII, aumenta a atividade do SNS do baço, gânglios linfáticos e da medula óssea, que por sua vez aumenta a atividade e liberação de linfócitos T circulantes e mediadores inflamatórios como IL-1, IL-2, IL-6 e IL-16 na circulação, resultando em disfunção endotelial e inflamação vascular (GANTA et al. 2005; HARRISON et al., 2011; SANTISTEBAN et al. 2013).

No sistema nervoso central, a AII atua particularmente no órgão subfornicial (OSF), órgão vasculoso da lâmina terminal (OVLT), área postrema (AP) e núcleo paraventricular do hipotálamo. A ação da angitensina II ao atuar no OSF e OVLT ativa as fibras pós-ganglionares simpáticas, e estas promovem a vasoconstrição periférica, aumento do inotropismo e liberação de renina nas células justaglomerulares. No núcleo paraventricular do hipotálamo a AII promove aumento da atividade simpática renal. No sistema nervoso periférico a AII atua nos gânglios simpáticos facilitando a liberação da acetilcolina, e nas terminações simpáticas cardíacas e vasculares facilitando a liberação de noradrenalina. Com a hiperatividade simpática induzida pela AII ocorre vasoconstrição, que provoca o aumento da resistência vascular periférica total, aumento da pós-carga e, subsequentemente, da PA (DURAND et al. 2012). A AII também é responsável por atenuar de forma importante o barroreflexo, agindo principalmente no núcleo do trato solitário (SANTOS et al., 2012).

Na zona glomerular da glândula adrenal a AII, promove a liberação da aldosterona, a qual possui ação nos túbulos renais induzindo a reabsorção de sódio e retenção de água, com significativo aumento do volume extracelular, com comprometimento sobre a regulação da PA (DURAND et al., 2012).

Estudos demonstraram que a aldosterona pode promover a liberação de mediadores inflamatórios, como a ciclo-oxigenase-2, proteína de atração de monócitos (MPC-1) e ICAM-1 (molécula de adesão intercelular), recrutando monócitos e macrófagos nos vasos sanguíneos do coração e dos rins, com conseqüente fibrose tecidual (BROWN, 2013; SCHIFFRIN, 2013).

A aldosterona contribui para a instalação de estresse oxidativo através do aumento da atividade de macrófagos nas células mesangiais, podócitos, células endoteliais do coração e da artéria aorta, bem como pelo aumento da atividade enzima NADPH, condições estas descritas que predispõe a disfunção endotelial (BROWN, 2013).

O estimulo da liberação da endotelina-1 pode ser induzido pela AII e pela aldosterona, e contribui para a fibrose do tecido cardíaco, devido ao aumento da síntese de colágeno pelos fibroblastos no coração (BROWN, 2013). A endotelina-1 por si só, trata-se de um importante 
mediador do processo inflamatório no sistema cardiovascular, pois está envolvida com a liberação de IL-6 e ICAM-1 (SAVOIA; SCHIFFRIN, 2006).

A ativação crônica do SRAA provoca proteinúria em decorrência de hipertensão e aumento da permeabilidade capilar glomerular, além da interferência na expressão de nefrina. $\mathrm{O}$ aumento de oferta de proteínas ao túbulo resulta em inflamação tubulointersticial (SHIMIZU, 2005; BROWN, 2013; CAMPBELL, 2013). Assim, a proteinúria constitui outro fator de risco importante para o paciente doente renal (SYME et al., 2007; ZATZ et al., 2012).

Buranakarl et al. (2008) avaliaram possíveis indicadores de estresse oxidativo renal e características dos eritrócitos em cães sadios e em cães azotêmicos, tendo concluído que os cães doentes renais apresentaram aumento dos marcadores de estresse oxidativo, principalmente o malondialdeído (MDA) urinário.

Galvão et al. (2013) estudaram a peroxidação lipídica como forma de estimar o comportamento das ERO em cães com DRC em diferentes estágios. Neste estudo, foi observado que nos cães com DRC ocorre aumento da peroxidação lipídida, cuja intensidade independe do estágio da doença.

A anemia em pacientes com DRC predispõe ao estresse oxidativo, pois as hemácias representam o principal componente de defesa antioxidante, por possuírem altas concentrações de enzimas (glutationa) capazes de metabolizar as espécies reativas do oxigênio. A hipóxia tecidual aumenta consideravelmente a produção das espécies reativas do oxigênio e o componente lipídico da membrana eritrocitária está também sujeito à agressão oxidativa. Os produtos desta lipoperoxidação podem induzir o estresse oxidativo intracelular, e na ocorrência de deficiência da defesa do sistema antioxidante ocorrerá a hemólise (FERREIRA; MATSUBARA, 1997). Nestas condições, ocorrem alterações no metabolismo celular, que pode promover a liberação de mediadores inflamatórios, e estes induzem as células inflamatórias a produção de espécies reativas do oxigênio e a instalação de um processo inflamatório tecidual local (FRY; McGAVIN, 2012).

Suliman et al. (2002) avaliaram as concentrações plasmáticas de glutationa em pacientes humanos renais crônicos com graus diferentes de anemia, sendo constatado que, quanto menor o hematócrito, maior a redução das concentrações de glutationa no interior das hemácias. Lustoza (2004) avaliou as concentrações plasmáticas de glutationa total, reduzida e oxidada, e a superóxido-dismutase em grupos de cães anêmicos com insuficiência renal crônica (IRC), e constatou ausência de diferença nas concentrações eritrocitárias da glutationa total e glutationa reduzida entre os grupos. Entretanto, houve aumento considerável nos valores das concentrações eritrocitárias de glutationa oxidada e da atividade enzimática eritrocitária superóxido-dismutase 
em cães com IRC, sendo assim os resultados obtidos sugerem aumento do estresse oxidativo nos cães estudados.

Em condições de inflamação em decorrência do estresse oxidativo no paciente com DRC pode ocorrer inibição da produção do hormônio eritropoetina, bem como o impedimento do crescimento de eritroblastos jovens e a apoptose de eritroblastos imaturos (FRY; McGAVIN, 2012).

O aumento da atividade da ERO pode comprometer a produção de NO nas células endoteliais, limitando sua disponibilidade, aumentando à resposta de vasoconstritores, principalmente da AII (LEE, 2008). Em estudos realizados foi demonstrado que a deficiência de NO na DRC é atribuída por diversos fatores, que resultam em vasoconstrição e consequentemente hipertensão glomerular, HAS, proteinúria e evolução do agravamento da função renal (MODLINGER et al., 2004).

\section{ÓXIDO NÍTRICO}

O NO é um radical livre, gasoso, inorgânico, que possui sete elétrons de nitrogênio e oito de oxigênio, possuindo um elétron desemparelhado (DUSSE et al., 2003). Trata-se de um radical abundante que age em uma variedade de processos biológicos, em especial, quando produzido pelas células endoteliais desempenha um papel significante no controle cardiovascular, tanto no controle da resistência vascular como na agregação plaquetária (FÖRSTEMANN; SESSA, 2011). Este radical livre, também possui a função de neurotransmissor e de regulador do sistema imune. Originalmente o NO foi identificado como fator relaxante derivado do endotélio ("endotlheliumderived relaxing factor": EDRF) sendo um potente vasodilatador, difundindo-se rapidamente entre as células e dentro delas (MONCADA et al., 1991; VASCONCELOS et al., 2007).

O NO é formado a partir do nitrogênio da guanidina presente na L-arginina, sob a ação catalítica da enzima NO-sintase (NOS), gerando na formação de NO e um co-produto, a Lcitrulina (BAYLIS, 2008; ZATZ et al., 2012). Sendo um radical livre, o NO reage rapidamente com o oxigênio tanto na fase gasosa como em solução aquosa, formando o gás dióxido de nitrogênio na forma gasosa $\left(\mathrm{NO}_{2}\right)$ ou nitrito $\left(\mathrm{NO}_{2^{-}}\right)$e nitrato $\left(\mathrm{NO}_{3^{-}}\right)$em solução, ambos podem ser dosados e podem estimar a produção de NO (ZATZ et al., 2012).

A detecção do NO em amostras biológicas apresenta um desafio, em função de sua pequena concentração e sua meia vida extremamente curta. Desse modo, a determinação deste composto pode ser obtida de forma indireta, através da dosagem plasmática ou urinária de $\mathrm{NO}_{2}$ - e $\mathrm{NO}_{3^{-}}$, utilizando-se metodologias complexas, como a quimioluminescência (DUSSE et al., 2003). 
Muitas funções fisiológicas no rim são atribuídas ao NO, as quais são: (a) regulação da hemodinâmica glomerular e da função tubuloglomerular, (b) participação na natriurese pressórica, (c) manutenção da perfusão medular, (d) inibição da reabsorção de sódio tubular, e (e) atua como modulador da atividade do sistema nervoso simpático (LEE, 2008).

A produção contínua de NO é indispensável à manutenção da homeostase do sistema circulatório. Existem três isoformas de NOS, que possuem funções distintas. A isoforma nNOS, comumente denominada de isoforma I ou $\beta$ NOS é encontrada no encéfalo, na medula espinhal, nos gânglios simpáticos, nas glândulas adrenais, nos neurônios nitrérgicos e em outras estruturas como células endoteliais de pulmões, útero e estômago, células da mácula densa do rim, células da ilhota pancreática e da musculatura esquelética. A isoforma eNOS, também denominada de isoforma III, está ligada à membrana das células endoteliais, bem como a adesão e agregação plaquetária. A eNOS pode ser encontrada em sinciciotrofoblastos, células epiteliais tubulares do rim, células intersticiais do cólon e hipocampo (MONCADA et al., 1994; DAVIS et al., 2013). A iNO ou isoforma NOSII é ativada a partir de alguns estímulos patológicos como: (a) lipopolissacarídeos bacterianos (LPS), (b) interleucina-1, (c) endotoxinas e (d) fator de necrose tumoral, e são independentes de íons de cálcio. Essa isoforma pode ser expressa em uma grande variedade de tipos celulares, incluindo macrófagos, linfócitos, neutrófilos, eosinófilos, células de Kupffer, hepatócitos e células endoteliais (ZATZ et al., 2012).

Inicialmente as isoformas eNOS e a nNOS foram caracterizadas como constitutivas, ou seja, eram expressas e produzidas rotineiramente, sem a necessidade de indução gênica. E a iNOS para ser expressa seria dependente de condições específicas, como em infecções. Entretanto, hoje é sabido que estas diferenças são menos delimitadas, pois a eNOS e a nNOS podem ser induzidas em determinadas circunstâncias, enquanto que a iNOS é expressa constitutivamente em vários tecidos (ZATZ et al., 2012).

Nos rins são descritos a presença da isoforma eNOS no endotélio vascular da alça de Henle ramo ascendente e ducto coletor, sendo encontrada também a isoforma nNOS na mácula densa, arteríola aferente, alça de Henle ramo ascendente, cápsula de Browman e ducto coletor. A expressão da isoforma iNOS tem sido associada à condições de inflamação (LEE, 2008).

Estudos realizados demonstraram que a deficiência de NO na doença DRC resulta em vasoconstrição e consequentemente hipertensão glomerular, HAS, proteinúria e evolução do comprometimento da função renal (MODLINGER et al., 2004; FÖRSTEMANN; SESSA, 2011; ZATZ et al., 2012). São atribuídas como causas prováveis da deficiência de NO na DRC: (a) limitações na disponibilidade do substrato (L-arginina), devido sua diminuição na biossíntese renal, (b) transporte diminuído de L-arginina em células endoteliais em pacientes urêmicos, (c) concentração elevada de inibidores da síntese de NO, em particular dimetil-argininas assimétricas 
(ADMA), (d) redução da massa renal (córtex renal), relacionado a uma lesão e (e) na DRC com comprometimento tubular ocorre como consequência uma diminuição na reabsorção da Larginina (BAYLIS, 2008). Outra causa implicada na diminuição da disponibilidade de NO tratase do estresse oxidativo, pois, as ERO podem atuar inibindo o NO, por reagir com a molécula de NO produzida, diminuindo a sua disponibilidade para as células, favorecendo os processos tromboembólicos, isto se relaciona-se também com a formação de $\mathrm{O}_{2}^{-}$(ROSENSON 2004; BAYLIS, 2008).

O estresse oxidativo nos vasos sanguíneos contribui para o desenvolvimento de doenças tromboembólicas. O NO produzido pela eNOS induz a produção da enzima SOD na camada muscular do vaso e extracelular, diminuindo o $\mathrm{O}_{2}{ }^{-}$disponível e, consequentemente, a produção de ONOO-. O NO também induz a síntese de ferritina, que se liga a íons ferro livre e previne a geração de $\mathrm{O}_{2}^{-}$. Na presença da placa ateroesclerótica, os macrófagos ativados produzem $\mathrm{O}_{2}{ }^{-}$, expressam iNOS e produzem NO. Desse modo, são produzidos ONOO- e $\mathrm{OH}^{-}$, comprometendo ainda mais a integridade tissular, favorecendo a ativação da coagulação e contribuindo para obstrução da luz vascular (FÖRSTEMANN; SESSA, 2011).

\section{CONSIDERAÇÕES FINAIS}

A incapacidade renal de executar adequadamente suas funções de manutenção do equilíbrio hidroeletrolítico e ácido-base, excreção de catabólitos e regulação hormonal, estão associadas com o estresse oxidativo. A literatura consultada reporta o envolvimento das ERO nos mais diferentes transtornos sistêmicos ocasionados pela DRC. O estudo aprofundado destas substâncias desenvolve novas pesquisas para o diagnóstico precoce do comprometimento renal, bem como pesquisas em opções terapêuticas que possam atenuar os efeitos deletérios promovidos pelas ERO durante a progressão da DRC.

\section{REFERÊNCIAS}

BARBOSA, K. B. R.et al. Estresse oxidativo: conceito, implicações e fatores modulatórios. Revista de Nutrição, Campinas, v. 23, n. 4, p. 629-643, 2010.

BAYLIS, C. Nitric oxide deficiency in chronic kidney disease. American Journal of Physiology - Renal Physiology, Bethesda, v. 294, n. 1, p. F1-F9, 2008. fibrosis. Nature reviews practice - Nephrology, London, v. 9, n. 8, p. 459-468, 2013. 
BURANAKARL, C.et al. Relationships between oxidative stress markers and red blood cell characteristics in renal azotemic dogs. Research in Veterinary Science, London, v. 10, n. 2 p. 1010-103, 2008.

CACHOFEIRO, V.et al. Oxidative stress and inflammation, a link between chronic kidney disease and cardiovascular disease. Kidney International, New York, v. 111, n. 74, p. 54-59, 2008 .

CAMPBELL, D. J. Evolving concepts of the renin - angiotensina system: Do intravenous and subcutaneous angiotensina II increased blood pressure by different mechanisms? Clinical and experimental pharmacology and physiology, Oxford, v. 40, n. 8, p. 560-570, 2013.

DAVIS, D.; W.et al. Age dependent expression and distribution of nitric oxide (NO) synthase isoforms in the ovine kidney. Open Journal of Molecular and Integrative Physiology, Hubei, v. 3, n. 2, p. 61-70, 2013.

DURAND, M., T.; FAZAN, R.; SALGADO, H. C. Fisiologia da Pressão Areterial Normal. In: BRANDÃO, A. A.; AMODEO, C.; NOBRE, F. Hipertensão. $2^{\circ}$ ed. Rio de Janeiro: Elsevier, 2012, cap, 4, p. 29-39, 511p.

DUSSE, L., M. S.; VIEIRA, L. M.; CARVALHO, M. G. Revisão sobre óxido nítrico. Jornal Brasileiro de Patologia e Medicina Laboratorial, Rio de Janeiro, v. 39, n. 4, p. 343-350, 2003.

FERREIRA, A. L. A.; MATSUBARA, L. S. Radicais livres: conceitos, doenças relacionadas, sistema de defesa e estresse oxidativo. Revista da Associação Médica Brasileira, São Paulo, v. 43, n. 1, p. 61-68, 1997.

GALLE, J. Oxidative stress in chronic renal failure. Nephrology Dialysis Transplantation, Oxford, v. 16, n. 11, p. 233-235, 2001.

GALVÃO, A. L. B.et al. Mensuração da peroxidação lipídica em cães clinicamente estáveis nos diferentes estágios da doença renal crônica naturalmente adquirida. Revista de Educação

Continuada em Medicina Veterinária e Zootecnia, São Paulo, v. 11, n. 2, p. 86, 2013.

FRY, M. M.; McGAVIN, D. Bone Marrow, Blood Cells, and the lymphatic system. In: McGAVIN, M. D.;ZACHARY, J. F. Pathologic basis of veterinary disease. $5^{\circ}$ ed. St. Louis: Elsevier, 2012, p. 698-770, 1322p.

FÖRSTERMANN, U.; SESSA, W. Nitric oxide synthases: regulation and function. European Heart Journal, Oxford, v. 31, n. 33, p. 829-837, 2011.

GANTA, C. K.et al. Central angiotensina II-enhanced splenic cytokine gene expression in mediated by the sympathetic nervous system. American Journal Physiology. Heart and Circulatory Physiology, Bethesda, v. 58, n. 4, p. H1683-H1691, 2005.

LEE, J. U. Nitric oxide in kidney: Its physiological role and pathophysiological implications. Electrolyte \& Blood Pressure, Korean, v. 6, n. 27, p. 27-34, 2008.

LOCATELLI, F.et al. Oxidative stress in end-stage renal disease: an emerging threat to pacient outcome. Nephrology Dialysis Transplantation, Oxford, v. 18, n. 7, p. 1272-1280, 2003. 
LUSTOZA, M. D. Avaliação do estresse oxidativo em cães com insuficiência renal crônica e anemia. 2004. 96 f. Dissertação de mestrado em medicina veterinária - Universidade de São Paulo. Faculdade de Medicina Veterinária e Zooctenia, São Paulo, 2004.

MODLINGER, P. S.; WILCOX, C. S.; ASLAM, S. Nitric oxide, oxidative stress, and progression of chronic renal failure. Seminars in Nephrology, New York, v. 24, n. 4, 2004.

MONCADA, S. PALMER, R, M.; HIGGS, E. A. Nitric oxide: physiology, pathophysiology, and pharmacology. Journal of pharmacology and experimental therapeutics, Bethesda, v. n. 43, n. 2, p. 109-142, 1991.

MONCADA, S. Physiological effects of nitric oxide. Journal of the Hypertension, London, v. 12, n. 3, p. 35-39, 1994.

SANTISTEBAN, M. M.et al. Dysfunction brain-bone marrow communication: a paradigm shift in the pathophysiology of Hypertension, Current Opinion in Nephrology and Hypertension, London, v. 15, p. 377-389, 2013.

SANTOS, R. A. S.; FERREIRA, A. J.; PINHEIRO, S. V. B. Papel dos principais componentes na gênese da hipertensão arterial: sistema renina-angiotensina. In: BRANDÃO, A. A.;

AMODEO, C.; NOBRE, F. Hipertensão. $2^{\circ}$ ed. Rio de Janeiro: Elsevier, 2012, cap, 8, p. 61-70, $511 \mathrm{p}$.

SAVOIA, C.; SCHIFFRIN, E. Inflammation in hypertension, Current Opinion in Nephrology and Hypertension, London, v. 15, p. 152-158, 2006.

SCHIFFRIN, E. L. The immune system: role in hypertension. The Canadian Journal of Cardiology, Oxford, v. 29, n. 5, p. 543-548, 2013.

SCOTT, A. N. D. Oxidative stress and chronic kidney disease. Veterinary Clinics of North American Small Animal Practice, New York, v. 38, n. 1, p. 157-166, 2008.

SULIMAN, M. E.et alInfluence of nutritional status on plasma and erythrocyte sulphur amino acids, sulph-hydryls, and inorganic sulphate in end-stage renal disease. Nephrology Dialysis Transplantation, Oxford, v. 17, n. 6, p. 1050-1056, 2002.

SHIMIZU, M. H. M. A N-acetilcisteína atenua a progressão da doença renal crônica. Tese de doutorado em medicina - Universidade de São Paulo USP. 2005. 105p. Faculdade de Medicina Ribeirão Preto. Departamento de Clínica Médica, Ribeirão Preto, 2005.

SYME, H., M.; FLETCHER, M., G., R.; BAILEY, S., R.; ELIOTT, J. Measurement of aldosterone in feline canine and human urine. The Journal of Small Animal Practice, Oxford, v. 48, n.4, p. 202-208, 2007.

ROSENSON, R. S. Statin in atherosclerosis: lipid - lowering agents with antioxidant capabilities. Journal of Atherosclerosis Research, Amsterdam, v.173, n. 1, p. 1-12, 2004.

VASCONCELOS, S. M. L.et al. Espécies reativas do oxigênio e de nitrogênio, antioxidantes e marcadores de dano oxidativo em sangue humano: principais métodos analíticos para sua determinação. Química Nova, São Paulo, v. 30, n. 5, p. 1323-1338, 2007. 
URSO, C.; CAIMI, G. Oxidative stress and endothelial dysfunction. Minerva Medicina, Torino, v. 102, n. 1, p. 59-77, 2011.

WILCOX, C. S.; TISHER, C. C. Manual de Nefrologia e Hipertensão. São Paulo: Teccmed, 2008.

ZAFARULLAH, M.et al. Molecular mechanisms of $\mathrm{N}$-acetylcysteine actions. Cellular and Molecular Life Sciences. New York, v. 60, n.1, p. 6 - 20, 2003.

ZATZ, R.; SEGURO, A. C.; MALNIC, G. Bases Fisiológicas da Nefrologia. São Paulo: Atheneu, 2012. 394 